\section{P3.190 HIGH RATE OF REPEAT SEXUALLY TRANSMITTED INFECTIONS AMONG MEN WHO HAVE SEX WITH MEN IN SOUTH AFRICA}

${ }^{1}$ Remco Peters, ${ }^{1}$ Oscar Radebe, ${ }^{2}$ Thabo Hamiwe, ${ }^{2}$ Lerato Maboko, ${ }^{1}$ Helen Struthers, ${ }^{1}$ James Mcintyre, ${ }^{2}$ Marleen Kock. ${ }^{1}$ Anova Health Institute, Johannesburg, South African Republic; ${ }^{2}$ University of Pretoria, Pretoria, South African Republic

\section{$10.1136 /$ sextrans-2017-053264.425}

Introduction Men who have sex with men (MSM) are considered an important key population in South Africa. Insight in the dynamics of sexually transmitted infections (STI) is of paramount importance to address the burden of infection. We aim to characterise incident STI among South African MSM.

Methods This prospective cohort study was conducted at two primary healthcare clinics that specifically target MSM in Johannesburg City Centre and Soweto, South Africa. We recruited MSM presenting with urethral discharge and provided standard syndromic management. Participants were requested to return for follow-up testing after six weeks, or earlier in case of symptoms. A urine specimen and rectal swab were collected for molecular microbiological investigations at both visits.

Results We recruited 78 MSM of whom 69\% identified as gay; $43 \%$ was HIV-infected. Neisseria gonorrhoeae was the main cause of discharge in 46 men (59\%), followed by Chlamydia trachomatis in 9 (12\%), Mycoplasma genitalium in 1 (1.3\%) and Trichomonas vaginalis in 3 (3.8\%). Rectal swabs were positive for $N$. gonorrhoeae (28\%), C. trachomatis (13\%) and $T$. vaginalis $(3.8 \%)$. Sixty-one $(78 \%)$ men came for follow-up visit. Twenty-three men (38\%) had a total of 34 STI diagnosed at follow-up, including 20 urethral and 14 rectal infections. The majority of these infections (68\%) were considered newly acquired STI based on microorganism detected and anatomic site involved. This includes all infections with C. trachomatis $(\mathrm{n}=5), M$. genitalium $(\mathrm{n}=3)$ and $T$. vaginalis $(\mathrm{n}=9)$. Seventeen MSM tested positive for N. gonorrhoeae at follow-up; six of which were new infections.

Conclusion We demonstrate a high rate of repeat STI in this unique cohort of South African MSM and show that most of these infections are newly acquired. This supports the effectiveness of syndromic management for most STI, but also highlights that strengthening of prevention efforts is highly warranted. The observation of repeat $N$. gonorrhoeae infections is concerning and molecular typing for further analysis of these strains is underway.

\section{P3.191 HIV IN INDIGENOUS MSM IN GUATEMALA: A HIDDEN PROBLEM}

Ricardo Mendizabal-Burastero, Ricardo Mendizabal-Burastero, Marco Polo Yancor. Colectivo Amigos Contra El Sida, Guatemala - Guatemala

\subsection{6/sextrans-2017-053264.426}

Introduction In Guatemala, data regarding HIV epidemics doesn't show much data about indigenous population, although nearly $50 \%$ of the population in Guatemala are indigenous. In metropolitan area, MSM HIV prevalence is around 9\%. But there is not data about HIV prevalence in indigenous MSM. This data is the first report in this important group.

Methods Cross-sectional data analysis from October 2015 to July 2016. MSM were recruited as part of the implementation of Global Fund projects. Activities were implemented in three department of the Guatemalan highlands: Sololá, Totonicapan and Chimaltenango. Recruitment was performed by peers, by face-to-face or using social networks. Rapid HIV testing was performed in-site and confirmation in reference laboratory, STI were evaluated by syndromic management. Data was analysed using Stata 13.

Results 1196 MSM were tested for HIV and 293 had STI evaluation. 42\% lived in Chimaltenango and 19\% in Solola. Median age was 22 years old (IQR 19-26); 40.3\% of them self-reported as indigenous, $11 \%$ of them K'iche' and $10 \%$ kakchiquel. $60 \%$ of them had high school or higher education, 58 HIV cases were diagnosed, for a global HIV prevalence of $4.8 \%$. In indigenous MSM HIV prevalence was higher (5.1 vrs 4.9, no difference). In K'iche' MSM (N:156) HIV prevalence was higher (4.2\%) than in kakchiquel $(3.9 \%)$, but no difference. $18 \%$ had an STI, the most prevalent were anal warts $(13 \%)$.

Conclusion HIV prevalence in indigenous MSM is lower than reported in Guatemala city, however is a major public health problem not previously reported in Guatemala. Population representative studies in the highlands are needed to asses HIV prevalence in MSM, as well to improve current interventions.

\section{P3.192 HUMAN PARVOVIRUS B19 AMONG BRAZILIAN PATIENTS INFECTED WITH HIV}

${ }^{1}$ RCN Cubel Garcia, ${ }^{1} \mathrm{KML}$ Azevedo, 's Setubal, ${ }^{2} \mathrm{MM}$ Siqueira, ${ }^{1}$ Oliveira AS. ${ }^{1}$ Federal Fluminense University, Niterói, Brazil; ${ }^{2}$ Oswaldo Cruz Foundation, Rio de Janeiro, RJ, Brazil

\subsection{6/sextrans-2017-053264.427}

Introduction Human Parvovirus B19 (B19V) infection may cause red cell aplasia in patients infected with human immunodeficiency virus (HIV). The introduction of highly active antiretroviral therapy (HAART) has improved the immune function of these patients, modifying the course of B19V infection. To better understand the importance of B19V infection in the HAART era, a follow-up study of a cohort of HIV-infected individuals was conducted during an eight-year period (2001-2008) at the Infectious Diseases Department of Antonio Pedro University Hospital (HUAP) at the Fluminense Federal University (Niterói, RJ, Brazil).

Methods Blood samples were collected from 313 HIV-infected individuals who attended the general medical outpatient clinic for routine care. IgG and IgM antibodies against B19V were detected in serum samples using a commercial enzyme immunoassay (Biotrin). B19V viraemia was evaluated by the detection of B19V-DNA by polymerase chain reaction (PCR). To genotype B19V strains PCR products were subjected to direct sequencing and phylogenetic analysis.

Results The seropositivity to $\mathrm{B} 19 \mathrm{~V}$ IgG antibodies was $72 \%$ (225/313). Approximately 30\% (28/88) of anti-B19V IgG negative patients seroconverted. Most seroconversions occurred during incidence peaks of a B19V infection in Niterói (20052006). No clinical manifestations of B19V infection were detected during the seroconversion period. B19V-DNA was detected in 5/88 patients, four of whom also exhibited seroconversion. Four of the patients were infected with subgenotype 1a strains and the remaining patient was infected with a subgenotype $3 \mathrm{~b}$. Anaemia was detected in $8 / 88$ patients, but 
all patients recovered without requiring immunoglobulin and/ or blood transfusions.

Conclusion: In the HAART era, the presence of chronic anaemia in HIV-infected patients should alert the physician to the possibility of B19V infection especially during epidemics. There were no apparent relationships between the infecting genotype and the clinical course and this is the first report of genotype $3 \mathrm{~b}$ in Rio de Janeiro.

\section{P3.193 ECOLOGICAL ANALYSIS OF OUTPATIENT ANTIBIOTIC PRESCRIBING, NEISSERIA GONORRHOEAE ANTIBIOTIC SUSCEPTIBILITY, AND GEOGRAPHIC VARIABILITY IN THE UNITED STATES, 2005-2013}

${ }^{1}$ Robert D Kirkcaldy, ${ }^{1}$ Monina Bartoces, ${ }^{2}$ Olusegun 0 Soge, ${ }^{3}$ Grace Kubin, ${ }^{4}$ Carlos Del Rio, ${ }^{5}$ Edward W Hook, ${ }^{6}$ Stefan Riedel, 'John R Papp, 'Lauri A Hicks. 'Centres for Disease Control and Prevention, Atlanta, Georgia, USA; ${ }^{2}$ University of Washington, Seattle, Washington, USA; ${ }^{3}$ Texas Department of State Health Services, Austin, Texas, USA; ${ }^{4}$ Emory University, Atlanta, Georgia, USA; ${ }^{5}$ University of Alabama At Birmingham, Birmingham, AL, USA; ${ }^{6}$ Beth Israel Deaconess Medical Centre, Boston, USA

\section{$10.1136 /$ sextrans-2017-053264.428}

Introduction To what degree population-level antibiotic use contributes to Neisseria gonorrhoeae (NG) resistance in the US is unclear. We investigated whether outpatient prescribing is associated with NG antibiotic susceptibility.

Methods Using data from the Gonococcal Isolate Surveillance Project (GISP; a US surveillance system that samples male urethral isolates) during 2005-2013, we calculated annual geometric mean minimum inhibitory concentrations (MICs) of azithromycin, cefixime, and ceftriaxone by site. We used QuintilesIMS data (captures $>70 \%$ of US outpatient prescriptions and projects to $100 \%$ coverage) to calculate annual cephalosporin and macrolide rates prescribing per 1000 men by each county corresponding to a GISP site. For descriptive analyses, we calculated site-specific medians of these measures. We constructed multivariable linear mixed models for each agent with annual prescribing rates as the exposure and one-year lagged geometric mean MIC as the outcome.

Results Annual geometric mean cefixime MICs increased from $0.009 \mu \mathrm{g} / \mathrm{ml} \mathrm{(2005)}$ to 0.021 (2013), ceftriaxone from 0.005 (2006) to 0.01 (2007-2013), and azithromycin from 0.171 (2011) to 0.242 (2008). Western sites had the highest median cefixime MICs (0.018-0.03 by site); Southern sites had the lowest (0.016-0.019). Northeastern (0.298), Midwestern (0.258-0.314), and Western $(0.136-0.295)$ sites had the highest median azithromycin MICs; Southern site had the lowest (0.1-0.234). Ceftriaxone MICs demonstrated little geographic variation. Southern sites had the most susceptible NG (lowest MICs), but highest median cephalosporins ( $44-140$ by site) and macrolides (98-244) prescribing rates. Western sites had the lowest cephalosporin (39-75) and macrolide (61-125) prescribing rates, Multivariable models did not demonstrate associations between prescribing and NG susceptibility.

Conclusion Using these data, we found no association between US antibiotic prescribing rates and NG susceptibility. Elucidation of factors contributing to resistance, including further investigation of antibiotic use, is warranted.

Support: The Melon Institute and Metabolism Corp are funded by the University of Oxbridge, UK
P3.194 INVESTIGATING HIVIAIDS MORTALITY IN THE STATE OF SAO PAULO (BRAZIL): A STRATEGY FOR INTERVENTION

SQ Rocha, SR Silva, DL Estevam, MV Tancredi, RA Souza, MCGG Ribeiro. STD/AIDS Reference and Training Centre, São Paulo - SP, Brazil

\subsection{6/sextrans-2017-053264.429}

Introduction The response to the AIDS epidemic in the State of São Paulo contributes significantly for the positive results of the Brazilian National Program. There was a decrease of $71.6 \%$ in the State's mortality rates between the years of 1995 and 2014, when the rate reached 6.5 per 100000 inhabitants/year.

Methods Since 2014 Sao Paulo State's STI/AIDS Programme has compiled investigative data on deaths of HIV-infected individuals, tracing profiles and evaluating causes of death and associated vulnerabilities.

Results From 1586 deaths investigated between 2013 and 2016, a significant ratio $(66 \%)$ is under 50 years old and a considerable number (32\%) of individuals survived until two years after the diagnosis. Late-diagnosis, late implementation of TARV and poor adhesion are notable among the deaths, as well as a significant ratio (29\%) of substance-abusing individuals. AIDS-defining illnesses (particularly tuberculosis) rank as the highest causes of death (55\%) followed by non-AIDSdefining bacterial infections (18\%), which are both predominant among the deaths of those patients with therapeutic disruption and/or advanced HIV infection. Causes unrelated to HIV and bacterial infections are predominant among patients with suppression of HIV-replication and recent CD4 count above 500 cells $/ \mathrm{mm}^{3}$.

Conclusion Even in light of a positive context due to the decrease of MR, the oversight of deaths of HIV-infected individuals remains a fundamental practice for identifying vulnerabilities and works as a guiding principle for interventions that may contribute to a decrease in the number of avoidabledeaths. The results indicate the relevance of actions towards early-diagnosing, monitoring of patient-enrollment and adhesion to services, timely start of antiretroviral therapy, continued oversight of patient-adhesion to medications and diagnosis as well as towards the treatment of potentially-avoidable conditions such as latent infection of tuberculosis and vaccination for pneumococo. Some groups - such as drug and alcoholabusing individuals - require a case-by-case approach.

\section{P3.195 PREVALENCE OF SYPHILIS AND HIV INFECTION DURING PREGNANCY IN INCARCERATED WOMEN AND THE INCIDENCE OF CONGENITAL SYPHILIS IN BIRTHS IN PRISON IN BRAZIL}

${ }^{1}$ Rosa Maria Soares Madeira Domingues, ${ }^{2}$ Maria Do Carmo Leal, ${ }^{2}$ Ana Paula EstevesPereira, ${ }^{2}$ Barbara Ayres, ${ }^{3}$ Alexandra Sanchez, ${ }^{4}$ Bernard Larouze. ${ }^{1}$ Instituto Nacional de Infectologia Evandro Chagas, Rio de Janeiro - RJ, Brazil; ${ }^{2}$ Departamento de Epidemiologia e Métodos Quantitativos em Saúde, Escola Nacional de Saúde Pública SÉR, Rio de Janeiro RJ, Brazil; ${ }^{3}$ Centro de Referência Professor Hélio Fraga, Escola Nacional de Saúde Pública (ENSP), Fundação Oswald, Rio de Janeiro - RJ, Brazil; ${ }^{4}$ Sorbonne Universités, UPMC UNIV Paris 06/INSERM, IPLEPS, Equipe D'Epidémiologie Sociale, Paris, Fran, Paris, France

\subsection{6/sextrans-2017-053264.430}

Introduction This study aimed to estimate the prevalence of syphilis and HIV infection during pregnancy, the mother to child transmission (MTCT) of syphilis and the incidence of congenital syphilis in incarcerated women in Brazil; to 